\title{
Determination of relative dissociation energy from electro-optics as a single-proposed new parameter of vegetable oil quality
}

\author{
Ketut Sofjan Firdausi ${ }^{1}$, Izzah Afiefah ${ }^{2}$, Heri Sugito ${ }^{1}$, Ririn Widya Septianti ${ }^{1}$, Very Richardina ${ }^{1}$, \\ Qidir Maulana Binu Soesanto ${ }^{1}$, and Much. Azam ${ }^{1}$ \\ ${ }^{1}$ Department of Physics, Diponegoro University, Semarang 50275, Indonesia; k.sofjanfirdausi@yahoo.co.id \\ 2Undergraduate Program, Department of Physics, Diponegoro University, Semarang 50275, Indonesia; izzahafiefah@st.fisika.undip.ac.id
}

\section{A R T I C LE IN F O}

\section{Article history:}

Received : 20 June 2019

Accepted : 2 November 2019

Available online : 30 November 2019

\section{Keywords:}

Relative dissociation energy

Electro-optics

Vegetable oil quality

\section{A B S T R A C T}

In this report, we determine the relative dissociation energy as a single new proposed parameter of oil quality through changes of light polarization of the electro-optics effect on cooking oil. The sample was canola oil which had been heated in 0.5 hours, 2 hours and 4 hours. The light source used in the experiment was a green pointer laser with a wavelength of $532 \mathrm{~nm}$. The sample was applied to a potential difference from 0 to $9 \mathrm{kV}$ to obtain the electro-optics effect in form of the change of the polarization angle $\Delta \theta$ as function of potential difference $\Delta V$. The relative dissociation energy was obtained from the fitting data of the relative Lenard-Jones potential energy curves represented by change of polarization per unit change of potential difference, $\Delta \theta / \Delta V$. The result shows that the relative Lenard-Jones potential energy as function of relative molecular distance fit with the experimental data of $\Delta \theta / \Delta V$. The relative dissociation energy is reduced as the canola oil quality decreased after heated. The relative dissociation energy provides simple physical understanding about electro-optics effect on cooking oils. The study of electro-optics polarization that represents relative LenardJones potential energy is a new but somewhat intuitive, which can be further improved and emphasized for grouping, mapping, and determining various cooking oil quality and halal food due to the lard contamination.

\section{Introduction}

Electro-optics effect is a change of optical characteristics of sample because it applies an additional external electric field on the sample. The electro-optics is also known as Kerr-effect or Pockel effect which measures the change of refractive index of sample (crystal or liquid) due to an external electric field.

According to the best of our knowledge, there are only a few electro-optics reports that measure polarization changes directly to a sample, especially in cooking oil. In our previous reports, we have found that by using electro-optics effect on cooking oils [1-3], it can increase the change of polarization angle or well-known as the change of optical activity.

Unfortunately, the electro-optics so far has not been recognized as one of a parameter for standard evaluation of cooking oil quality. As we have found that the change of electro-optics polarization provides cooking oil quality indicator and for certain purposes it is very useful for examination of preliminary quality [1-2, 4-6]. In our previous works[1, 2], the physical variable represents the quality indicator is the change of polarization angle in ${ }^{\circ}$ or electro-optics gradient in $\% / \mathrm{kV}$, which is indicated by increasing saturated fatty acids or decreasing polyunsaturated fatty acids in oil sample. For preliminary test of oil quality, the change of electro-optics polarization seems powerful in comparison to the other methods $[1,4,6]$.

However, in our consideration, the important physical phenomenon for examination of quality does not base on the change of polarization, which indicates only the end result of direct measurement. In previous idea [7], we proposed that the important physical phenomenon should represent the fundamental physical process and this parameter should be regarded and hypothesized as single physical parameter of cooking oil quality. Based on this hypothesis, all behavior that changes the oil quality can be denoted only by the interaction between triglyceride (TG) molecules, which it obeys Van der Waals interaction. The applied external potential difference $\Delta V$ on sample hypothetically change the relative molecular distance and therefore change its relative Lenard-Jones potential energy. The light polarization changes per unit potential difference $\Delta \theta / \Delta V$ theoretically are proportional to 
relative Lenard-Jones potential energy and leads finally to relative dissociation energy, $\varepsilon_{r}$, as a new proposed single parameter of oil quality. In this report, we determine the relative dissociation energy through the electro-optics effect and verify it on canola oil as a special case.

\section{Method}

The sample was new canola oil at the time of the data acquisition and had relative different quality after heated in 30 minutes, 2 hours and 4 hours. For validation and sample characterization, all samples had been tested through GCMS instrument showing the highest composition of monounsaturated fatty acids more than $75 \%$. Table 1 show the samples used in the experiment.

Table 1: Sample of canola oil

\begin{tabular}{cc}
\hline sample & remark \\
\hline C1 & New canola oil \\
C2 & after heated in 30 minutes \\
C3 & after heated in 2 hours \\
C4 & after heated in 4 hours \\
\hline
\end{tabular}

The standard experimental procedure of electrooptics polarization was referring to the Firdausi et al [1]. Fig. 1 shows the measuring polarization changes of electric field $\mathbf{E}_{\mathbf{L}}$ of incoming light as external potential difference $\Delta V$ applied on sample.

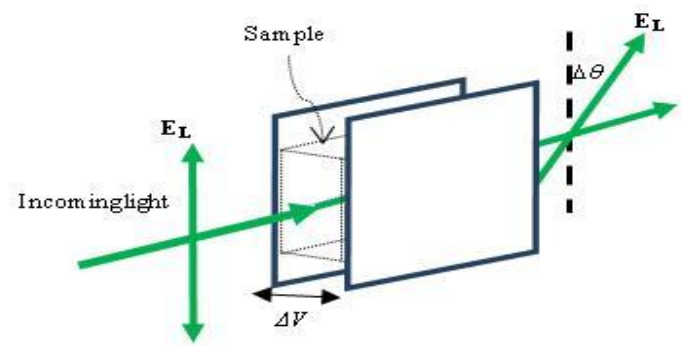

Fig. 1: The scheme of polarization angle $\Delta \theta$ measurement as potential difference $\Delta V$ applied on inserted sample in parallel plates.

The light source was a green pointer laser with $\lambda=532 \mathrm{~nm}$, and was adjusted to be linearly polarized using a polarizer. The sample was inserted between two parallel plates and was applied at high voltage $\Delta V$ from 0 to $9 \mathrm{kV}$. The change of the transmission polarization angle $\Delta \theta$ was measured by using an analyzer to obtain the relation of $\Delta \theta$ vs. $\Delta V$, for each of sample.

The determination of relative dissociation energy is explained as follows. In parallel plates with separation $d$ and applied voltage $\Delta V$, the external electric field that crosses the plates is $E=\Delta V / d$, in which the TG molecules will be polar and become induced electric dipoles. For a given $d$, we can alter the electric fields by changing $\Delta V$. This potential difference supposedly gives effective molecular distance through the linear relation $\xi \sim \Delta V$, where $\xi$ is dimensionless scale factor representing relative molecular distance. When $\xi$ increases due to the increasing $\Delta V$ and then it leads to the change of Lenard-Jones potential energy $E_{\mathrm{Lj}}$. In actual condition, the Lenard-Jones potential energy as function of actual molecular distance $R$ generally can be written as

$$
E_{L J}=\varepsilon\left[\left(\frac{R_{m}}{R}\right)^{12}-2\left(\frac{R_{m}}{R}\right)^{6}\right]
$$

The variable $R_{m}$ is the distance between two TG molecules when $E_{\mathrm{LJ}}$ reaches its minimum value. The important parameter of $\varepsilon$ is known as the dissociation energy when two TG molecules are separated at infinite distance, which is in the experiment to be obtained through the following explanations.

When a linear polarized light with field vector $\mathbf{E}_{\mathbf{L}}$ interacts with a dipole of induced TG molecules, $\mathbf{p}$, it creates an electrostatic potential energy $\mathbf{p} \bullet \mathbf{E}_{\mathbf{L}}$ which is proportional to potential energy $E_{\mathrm{LJ}}$. Experimentally, the value of $\mathbf{p} \bullet \mathbf{E}_{\mathbf{L}}$ is measured through the polarization change, $\Delta \theta$. Hence, according to our hypothesis, $\Delta \theta$ should be related to the potential energy $E_{\mathrm{LJ}}$. The change of polarization angle per unit potential difference $(\Delta \theta / \Delta V)$ thus represents a relative potential energy $E_{\mathrm{r}}(\xi)$ (dimensionless). Fig. 2 describes a model of forming an electric dipole of $\mathbf{p}$ when subject to potential difference of $\Delta V$ and its interaction with light to produce relative potential energy of $\Delta \theta / \Delta V$.
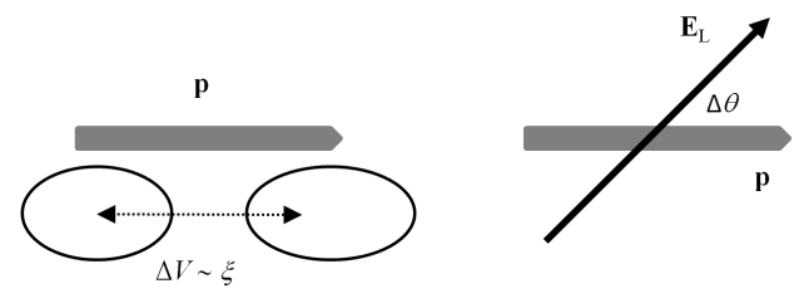

Fig. 2: A model of an induced dipole $\mathbf{p}$ from two closest TG molecules (left), and the interaction between a linear polarized light $\mathbf{E}_{\mathrm{L}}$ and dipole $\mathbf{p}$ (right)

We propose the relation of electro-optics polarization and the relative potential energy $E_{r} \sim \Delta \theta / \Delta V$ through the equation

$$
E_{r}=\beta \frac{\Delta \theta}{\Delta V}=\varepsilon_{r}\left\{\left(\frac{\xi_{m}}{\xi}\right)^{12}-2\left(\frac{\xi_{m}}{\xi}\right)^{6}\right\}
$$

The parameter $\beta$ is a proportional constant in unit $\mathrm{kV} /{ }^{\circ}$. The electro-optics parameter $\beta \Delta \theta / \Delta V \equiv E_{r}$ is relative potential energy, $\xi$ is relative molecular distance, and the value $\varepsilon_{r}$ is the desired relative dissociation energy. The values $\varepsilon_{r}$ and $\xi_{m}$ will be determined by using semi empirical formulas from experimental data $\Delta \theta / \Delta V$ as a function of $\xi$.

\section{Results and Discussion}

Fig. 3 shows the change of polarization angle $\Delta \theta$ as function of potential difference between plates $\Delta V$ for sample C1, C2, C3 and C4, respectively indicating a correspondence of good to low oil quality with the increasing $\Delta \theta$ from low to high value. 


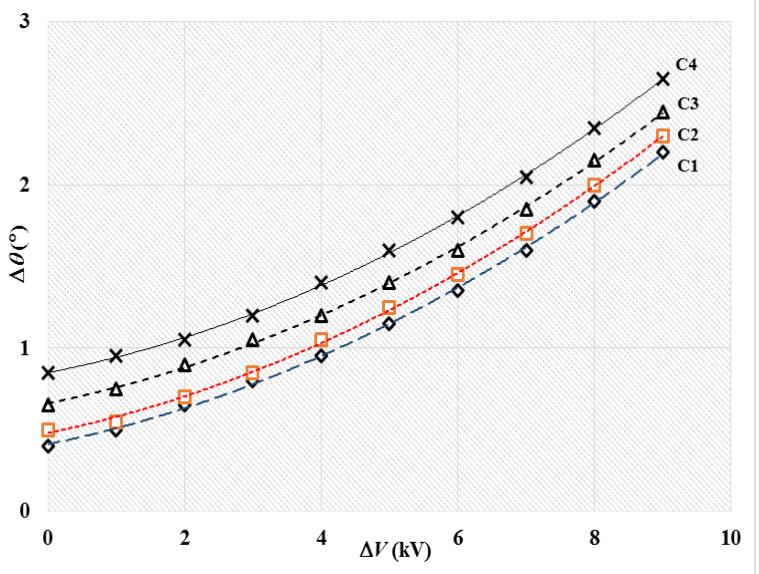

Fig. 3: The change of polarization angle $\Delta \theta$ as function of potential difference $\Delta V$ for sample $\mathrm{C} 1$, $\mathrm{C} 2, \mathrm{C} 3$ and C4.

The quadratic function between $\Delta \theta$ and $\Delta V$ is once again in agreement with our previous work [2-3], indicating non-linear responds of electrics polarization due to high potential difference on the sample. The increasing average polarization angle from $\mathrm{C} 1$ to $\mathrm{C} 4$ in fig. 3 describes the decreasing oil quality and provides important information of the developing saturated fatty acids and decreasing unsaturated fatty acids simultaneously, as reported also in our previous work [8]. The natural polarization (i.e. $\Delta \theta$ at $\Delta V=0$ at table 2) shows optical active characteristics of each sample and provides information of good or low oil quality indicated by small or high $\Delta \theta$, respectively. By increasing $\Delta V$, it produces more significant $\Delta \theta$ due to pure contribution of electro-optics effect for each sample and this shows how reliable the electrooptics polarization for preliminary test of cooking oil quality is.

If the values in fig. 3 are subtracted by their respective natural polarizations, we obtain pure electro-optics contribution for each sample as shown in fig. 4 and the average electro-optics contributions are also listed in table 2 .

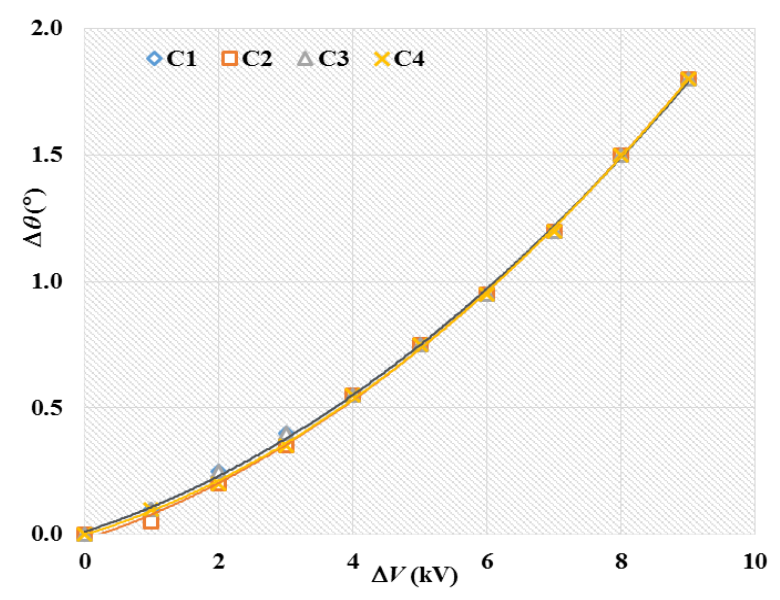

Fig. 4: The electro-optics contribution of polarization angle is equal shown by overlapping all curves for sample C1, C2, C3 and C4.
Table 2: Natural polarization $\Delta \theta_{\mathrm{N}}$ and average of electro-optics contribution $\Delta \theta_{\mathrm{E}}$

\begin{tabular}{ccc}
\hline sample & $\Delta \theta_{\mathrm{N}}\left(^{\circ}\right)^{*}$ & $\Delta \theta_{\mathrm{E}}\left(^{\circ}\right)^{* *}$ \\
\hline $\mathrm{C} 1$ & $0.40 \pm 0.04$ & $0.75 \pm 0.04$ \\
$\mathrm{C} 2$ & $0.50 \pm 0.04$ & $0.74 \pm 0.04$ \\
$\mathrm{C} 3$ & $0.65 \pm 0.05$ & $0.75 \pm 0.04$ \\
$\mathrm{C} 4$ & $0.85 \pm 0.03$ & $0.74 \pm 0.04$ \\
\hline *natural polarization is the value $\Delta \theta$ at $\Delta V=0$ \\
** Average values of pure electro-optics contribution
\end{tabular}

It shows very important results that an equal electro-optics contribution of polarization angle occurs for all samples. It can be due that the duration of heating on sample did not influence significantly to the developing new fatty acids. And in addition to the canola oil itself it is still dominated by monounsaturated fatty acids more than $75 \%$, which in turn contributed only to the equal development of induced dipoles in all sample. This means that molecular interaction with electro-optics effect gives similar induced dipoles, because that the highest number of dominant fatty acids in canola oil provides identical electric dipole behavior, as it is already known that more than $75 \%$ in canola oil is monounsaturated fatty acids from the GCMS test result. For further investigation, it should be able also to map not only the relative quality but also various type of cooking oil. Yet, the pure polarization angle does not inform directly the fundamental process as a preliminary quality indicator. In the next paragraph, the important physical phenomenon that assumed as single quality parameter based on the molecular interaction will be discussed.

There are many parameters of vegetable oil quality namely physical and chemical parameters that cannot be measured simultaneously. If one or more parameters of quality are changed then it is caused mainly by existing other species, new molecules or atoms. According to our hypothesis, it will lead only to the interaction between molecules and will change merely to the different potential energy $E_{\mathrm{LJ}}$. Regarding to the equation (1), the only characteristics of potential energy is the dissociation energy of $\varepsilon$, and hence it describes as a single parameter that accumulating all change of physical or chemical parameters through Van der Waals interaction. A TG molecule can be regarded as a nonpolar molecule. The interaction between two closest TG molecules is similar to two neutral atoms. The value of $E_{\mathrm{LJ}}$ is influenced by the nuclear distance between two neutral adjacent atoms. In this circumstance, a non-polar TG molecule can be considered as a pseudo atom and the interaction is assumed as Van der Waals interaction that leads to the similar $E_{\mathrm{LJ}}$. If additional external electric field is applied, the TG molecules of sample will be polarized and changed the molecular distance $R$ between them. In average it changes the Lenard-Jones potential energy $E_{\mathrm{LJ}}$ as written in equation (1).The electrooptics configuration that develops the relative Lenard-Jones potential energy is similar to the strength of molecular interaction forces between two neutral TG molecules. The depth of the level energy $\varepsilon$ in $\mathrm{eV}$ from equation (1) in this case will be represented by relative dissociation energy $\varepsilon_{r}$ (dimensionless) from equation (2) as average 
relative dissociation energy of the entire molecular interaction and as a proposed new single parameter of the cooking oil quality.

Fig 5 shows the fitting experimental data of relative potential energy $\Delta \theta / \Delta V=E_{r}\left(\right.$ for $\beta=1 \mathrm{kV} /{ }^{\circ}$ ) against scale factor or relative molecular distance $\xi$ for sample C1, C2, C3 and C4. The experimental data of all sample well fit together with the curve model of Lenard-Jones potential energy, and it can be considered that the hypothesis has been experimentally proven for canola oil. All curves from fitting data in fig 5 have a common equation (assuming $\beta=1 \mathrm{kV} /{ }^{\circ}$ ) and is written as

$$
E_{r}=E_{0}+\varepsilon_{r}\left[\left(\frac{\xi_{m}}{\xi}\right)^{12}-2\left(\frac{\xi_{m}}{\xi}\right)^{6}\right]
$$
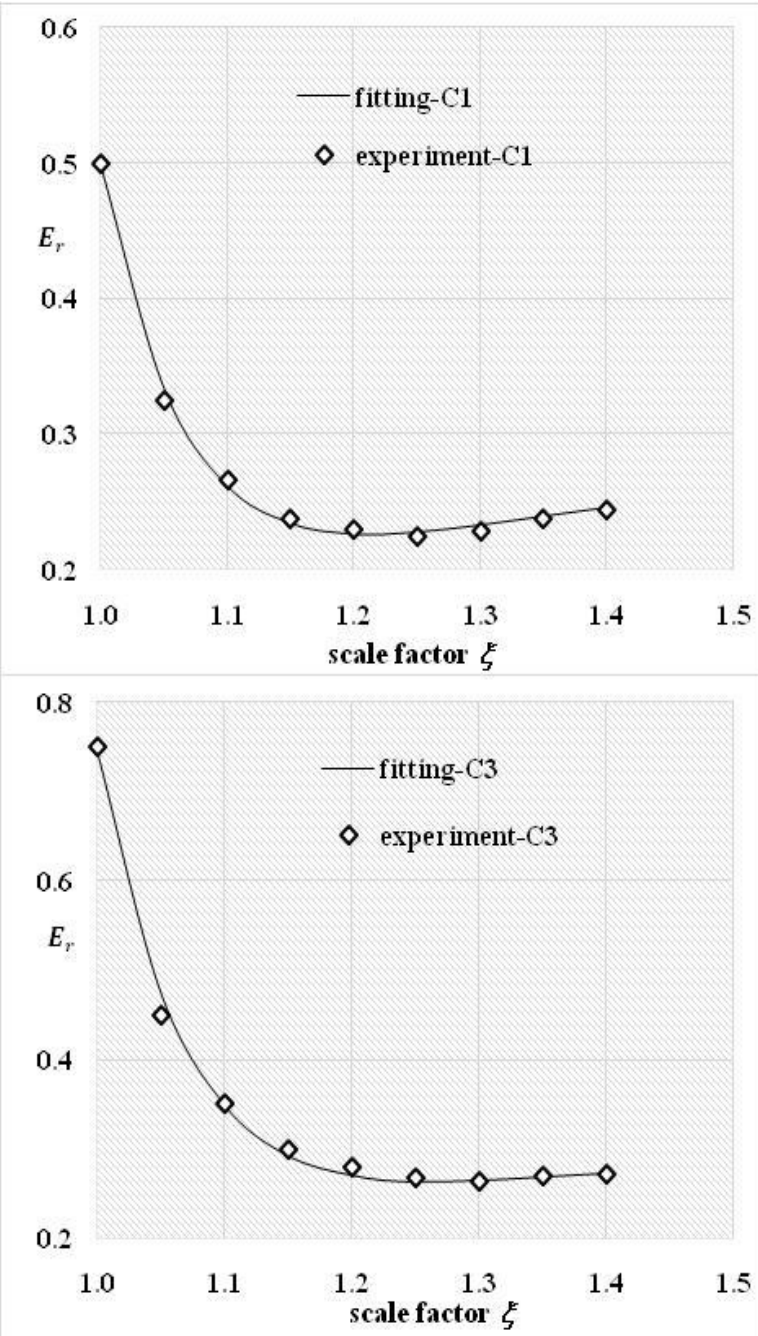

The relative dissociation energy from fig 6 is satisfied for $\varepsilon_{r 1}>\varepsilon_{r 2}>\varepsilon_{r 3}>\varepsilon_{r 4}$, which corresponds to the decreasing sample quality, shifting also their relative molecular distance for $\xi_{m 1}<\xi_{m 2}<\xi_{m 3}<\xi_{m 4}$ (in fig 6), respectively. These values are relatively compared only to canola oil between new oil and degraded oil quality. In the absence of external field (potential difference), because the natural optical activity of oil is relative small caused by existing small number of asymmetric TG molecules, therefore the reducing oil quality indicated by increasing natural polarization describes that there is more additional asymmetric TG molecules. It means that the increasing saturated fatty acids and reducing unsaturated fatty acids simultaneously during heating the sample leads to the increasing number of asymmetric TG molecules, which generally increases the natural polarization.
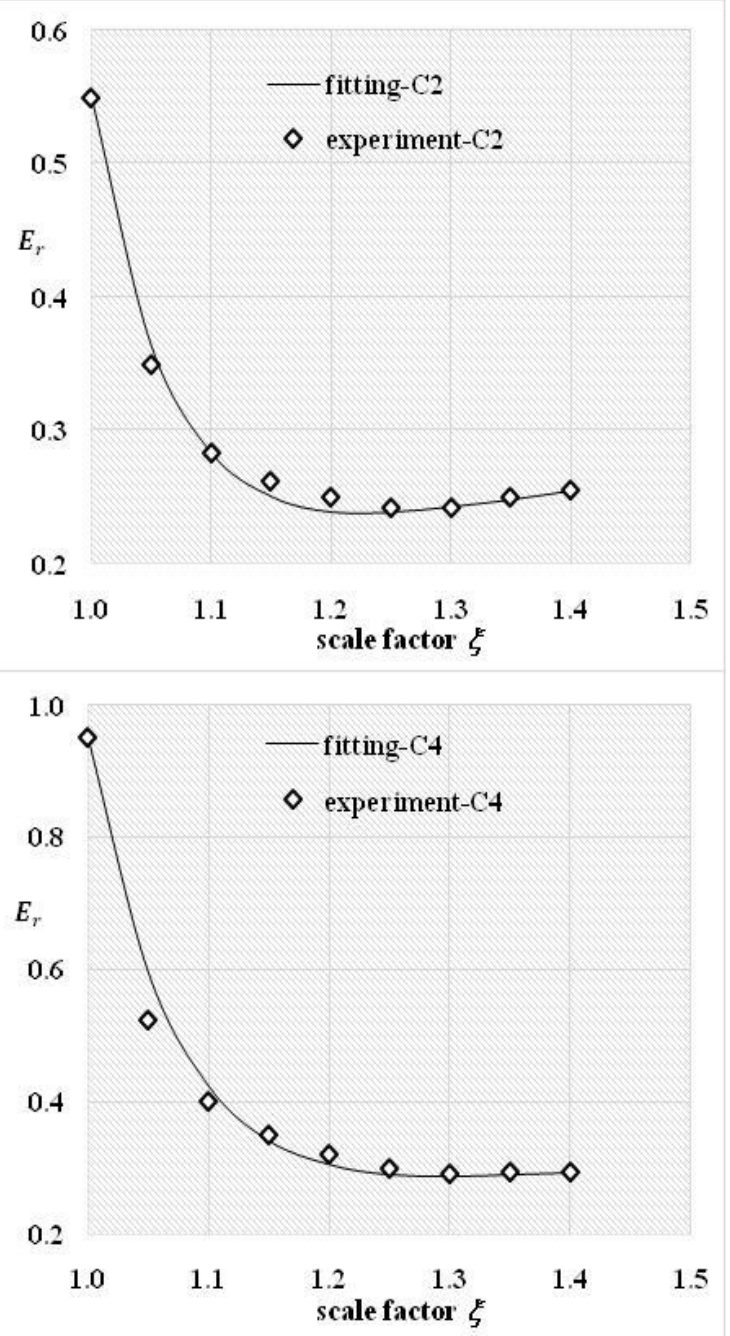

Fig.5:The fitting experimental data of relative Lenard-Jones potential energy for sample C1, C2, $\mathrm{C} 3$ and $\mathrm{C} 4$.

$E_{0}$ is the intercept value at axis $\Delta \theta / \Delta V$ when $\xi=$ $\xi_{m} / 2^{1 / 6}$ and as $\xi \rightarrow \infty$. The scale factor is also the relative molecular distance (dimensionless) and can be written as $\xi=c \Delta V+b$ as $\Delta V$ goes from 0 to $9 \mathrm{kV}$ with $c=0.05 \mathrm{kV}^{-1}$ and $b=0.95$. The equation (4) is also known as semi empirical formula in finding the values of $\varepsilon_{r}$ and $\xi_{m}$. The curves with the desired value of $\varepsilon_{r}$ for all samples are described in fig 6.The numerical values of the fitting data are presented in table 3.
Referring to the decreasing quality, which is accompanied by increasing asymmetric TG molecules, the molecular interaction force in asymmetric, should be less than in symmetric TG molecules. An asymmetric TG molecule is optically active whereas a symmetric TG molecule is optically inactive. This shows that a symmetric TG molecule provides stronger interaction of Van der Waals force than an asymmetric TG molecule. This fact makes a realistic physical explanation that in degraded canola oil with more existing asymmetric TG molecules shows more reducing Van der Waals force 
indicated by decreasing relative dissociation energy
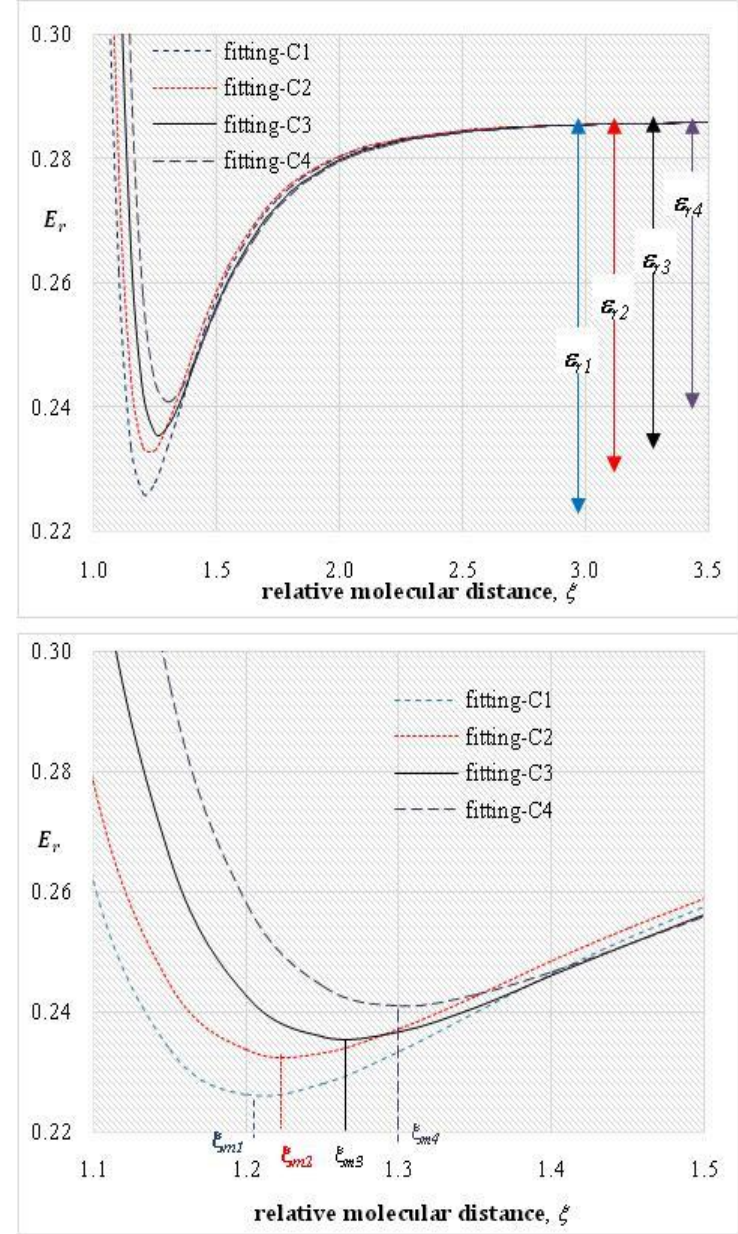

Fig. 6: Relative dissociation energy $\varepsilon_{r}$ and minimum molecular distance $\xi_{m}$ from fitting data.

of $\varepsilon_{\mathrm{r}}$. The minimum molecular distance operatively shifts to the higher distance after the developing asymmetric TG molecules in heated oil.
Lenard-Jones potential that is obtained from electrooptics consideration is rather intuitive and use physical understanding of interaction of matter with light. However the physical perspective in applying external electric fields on the samples results electric potential energy, which is in this case very possible represented by Lenard-Jones potential due to nonpolar TG molecules. For the next researches, the relative dissociation energy should be more comprehensively elaborated using formalism consideration from electrodynamics and quantum physics, and also should be applied for other various oils. The current standard parameters of cooking oil such as refractive index, free fatty acids, peroxide number, heavy elemental impurity, dielectric, water content and so on that could not be obtained simultaneously can now be very possible explained and replaced by single basic interaction of the sample's molecules. If one or more of the current standard parameters of quality is changed, so the reason is basically related to the change of molecular interaction of Lenard-Jones potential energy.

The recent methods [9-10] that could be related with electro-optics are dielectric constant. However, this method just only measures due to the increasing polar components in cooking oils after heating or during heating. This chemical aspect about polar molecules according to international standard is only up to $27 \%$ permitted in good oil quality, as mentioned by Lustosa et al [9]. Some other chemical aspects related to good oil quality according to National Standardization Agency of Indonesia [11] are such as peroxide number (not exceed than 10) and free fatty acids (not exceed than 0.6). The peroxide number is only related to the free radicals that often occur in cooking oil. And the other hand, the free fatty acids are simply fatty acids molecules that release form triglycerides in oils. These aspects i.e. polar components, free radicals, and free fatty

Table 3: The semi empirical formulas, the relative dissociation energy and relative minimum molecular distance obtained from fitting data of the curves in fig 4 , with setting $\beta=1 \mathrm{kV} /{ }^{\circ}$

\begin{tabular}{cccc}
\hline Canola & Semi empirical formulas & $\begin{array}{c}\text { Relative dissociation } \\
\text { energy, } \varepsilon_{r}\end{array}$ & $\begin{array}{c}\text { Relative minimum } \\
\text { molecular distance, } \xi_{\mathrm{m}}\end{array}$ \\
\hline $\mathrm{C} 1$ & $0.286+0.060\left[\left(\frac{1.21}{\xi}\right)^{12}-2\left(\frac{1.21}{\xi}\right)^{6}\right]$ & 0.060 & 1.21 \\
$\mathrm{C} 2$ & $0.286+0.054\left[\left(\frac{1.23}{\xi}\right)^{12}-2\left(\frac{1.23}{\xi}\right)^{6}\right]$ & 0.054 & 1.23 \\
$\mathrm{C} 3$ & $0.286+0.051\left[\left(\frac{1.27}{\xi}\right)^{12}-2\left(\frac{1.27}{\xi}\right)^{6}\right]$ & 0.051 & 1.27 \\
$\mathrm{C} 4$ & $0.286+0.045\left[\left(\frac{1.30}{\xi}\right)^{12}-2\left(\frac{1.30}{\xi}\right)^{6}\right]$ & 0.045 & 1.30 \\
\hline
\end{tabular}

The electro-optics effect that leads to LenardJones potential energy should be operative also to other cooking oils. And in the next study, the values of relative dissociation energy of canola oil obtained from semi empirical formula are still to be compared with other cooking oils. The benefit of canola oil based on the fact that the major component of monounsaturated fatty acids obtained from GCMS test is more than $75 \%$ of oil composition. So the interaction of TG molecules can be concentrated only on the dominant fatty acids composition. The relative dissociation energy including relative acids will definitely influence the whole Van der Waals interaction if one or all of them are changed. The change of the interaction leads to the change of single parameter, namely, the average Lenard-Jones potential energy, and it results back to the relative dissociation energy, which reflects a single parameter of quality.

The single parameter of oil quality is in this case able to be explained by fundamental interaction between TG molecules i.e. relative dissociation energy obtained from the curve of Lenard-Jones potential energy. The relative dissociation energy in the next study should be capable in grouping among 
the various types of oil, mapping between good oils and adulteration of oils, and determining halal oil due to lard contamination by using single parameter $\varepsilon_{r}$. Therefore comprehensively investigation related to the change of one or more from the standard parameters should be explained by the change of interaction that leads to the different Lenard-Jones potential for more various cooking oils. And more important, the actual energy $\varepsilon$ (in $\mathrm{eV}$ ) and molecular distance between TG molecules $R$ (in $\mathrm{nm}$ ) are still to be obtained and should be compared with other data spectroscopy. The useful of single proposed parameter $\varepsilon_{r}$ here is still open to be carefully discussed. In addition, the relative dissociation energy $\varepsilon_{r}$ can be widely developed particularly in bioscience.

\section{Conclusion}

The basic idea of the single quality parameter lays on the fact that the mutual interaction between two closest TG molecules will influence other physical variables namely refractive index, dielectric constant, and heavy element content and so on. This interaction leads finally to the relative Lenard-Jones potential energy, and can be measured when linearly polarized light is passed through the oil sample in the applied electro-optics condition.The electrooptics effect on canola oil is supposedly able to be used to explain and obtain relative dissociation energy describing single parameter of oil quality, and from the result we have shown that the experimental data fit with a model curve of relative Lenard-Jones potential energy. The semi empirical formula obtained from the fitting data results the relative value of dissociation energy and minimum relative distance between two molecules. The relative dissociation energy for a unit $\beta=1 \mathrm{kV} /{ }^{\circ}$ of the new canola oil measured in the experiment is 0.06 . This value is reduced when the oil quality is decreased, and at the same time the minimum relative molecular distance increases when the oil quality decreases. As a new single parameter of cooking oil quality proposed in this study, the relative dissociation energy should be emphasized for grouping, mapping, and determining various cooking oil quality and halal food due to the lard contamination in the next study. In addition, the relative dissociation energy obtained from the curve of Lenard-Jones potential energy provides simply understanding about interaction between TG molecules. The relative Lenard-Jones potential energy obtained from the electro-optics polarization change in this case is a new finding particularly for cooking oil and gives good opportunities in improving a simple and accurate method or instrument for developing food sciences. The absolute value of the dissociation energy and the absolute molecular distance in average between $\mathrm{TG}$ molecules are still interesting to be obtained through the comparison to the spectroscopy data. The basic molecular interaction of Lenard-Jones potential energy for cooking oils in this report can be further developed in biophysics, bioscience etc.
Acknowledgment

The research was supported by PTUPT scheme 2018 of Diponegoro University with contract number: 101-126/UN7.P4.3/PP/2018.

\section{References}

[1] K. S. Firdausi, H. Sugito, H. Rahmawati and A. B. Putranto, The relationship between electrooptics gradient and fatty acids composition in a new investigation on palm oil quality, Advanced Science Letters, 23, 6579-6581 (2017).

[2] K. S. Firdausi, K. Triyana and A. I. Susan, An improvement of new test method for determination of vegetable oil quality based on electro-optics parameter, Berkala Fisika, 15, 77 86 (2012).

[3] N. Widyastuti, M. Azam and K. S. Firdausi, Studi efek elektro-optis pada minyak goreng, Berkala Fisika, 12, 63-68 (2009).

[4] K. S. Firdausi and H. Rahmawati, Review of a simple powerful polarizer for testing of edible oil quality, Berkala Fisika, 18, 137-142 (2015).

[5] S. Murni, A. B. Putranto, E. Hidayanto dan K. S. Firdausi, Evaluasi kualitas beberapa jenis minyak goreng kemasan setelah dipanaskan menggunakan sifat elektrooptis, Berkala Fisika, 15, 119-122(2012).

[6] H. Sugito, K. S. Firdausi and N. K. Putri, Design of integrated polarizer for detection of lard impurities in cooking oil, Journal of Physics: Conference Series, 1025, 012008 (2018).

[7] K. S. Firdausi, H. Sugito, R. Amitasari, S. Murni and A. B. Putranto, Electro-optics effect as a new proposed method for determination of vegetable oil quality and a study of most responsible physical processes, Proceeding of International Seminar on New Paradigm and Innovation on Natural Science and Application, Semarang (2013).

[8] I. Afiefah, M. Azam, H. Sugito, K. S. Firdausi, Contribution of electro-optics effect on canola oil as a new alternative method for determination of oil quality using transmission and fluorescence polarization, Journal of Physics: Conference Series, 1217, 012030 (2019).

[9] K. A. S. S. Lustosa, A. M. P. Santos and E. J. P.Santos, System on chip for portable cooking oil quality determination, Proceeding of the 6th Ibero-American Congress on Sensors, Sao Paulo (2008).

[10] M. Pecovska-Gjorgjevich, A. Andonovski and J. Velevska, Dielectric constant and induced dipole moment of edible oils subjected to conventional heating, Macedonian Journal of Chemistry and Chemical Engineering, 31, 285294 (2012).

[11] National Standardization Agency of Indonesia, Minyak goreng, SNI 3741 (2013) 